\title{
Editorial: Virtual Reality Applications in Military and Veteran Medicine and Healthcare
}

\author{
Rudolph P. Darken ${ }^{1 *}$, Barry Peterson ${ }^{2}$ and Julie Gore ${ }^{3}$ \\ ${ }^{1}$ Naval Postgraduate School, Monterey, CA, United States, ${ }^{2}$ VA Sierra Nevada Health Care System, Reno, NV, United States, \\ ${ }^{3}$ Birkbeck, University of London, London, United Kingdom
}

Keywords: healthcare, medicine, military, veterans, training, therapy

Editorial on the Research Topic

Virtual Reality Applications in Military and Veteran Medicine and Healthcare

When the editorial team conceived of this Research Topic, we began by exploring the boundaries of the topic area and what makes these subjects interesting and important. Our initial perspective was that military and veterans healthcare is unique because many of the most severe conditions and injuries are sustained by military personnel and that many of these conditions (PTSD being a prime example) are lifelong conditions that must continue to be addressed long after leaving military service to sustain quality of life. Clearly, in this area of VR/XR research, the stakes are high. Help is needed, and there is a common belief that VR/XR has a role to play. What is unclear is what that role is. To what types of problem domains is VR/XR best suited? What are the best ways to apply the technology to the best possible result?

We expected to see a variety of prototypes and studies dealing with VR/XR applications to

OPEN ACCESS

Edited and reviewed by: Albert Rizzo, University of Southern California, United States

*Correspondence: Rudolph P. Darken darken@nps.edu

Specialty section:

This article was submitted to Virtual Reality in Medicine, a section of the journal Frontiers in Virtual Reality

Received: 18 January 2022 Accepted: 28 January 2022 Published: 17 February 2022

Citation: Darken RP, Peterson B and Gore J (2022) Editorial: Virtual Reality Applications in Military and Veteran

Medicine and Healthcare.

Front. Virtual Real. 3:856666. doi: 10.3389/frvir.2022.856666 military and veterans healthcare. We were not wrong, but we were not exactly right either.

In this Research Topic, you will find articles on important areas of healthcare for military and veterans, specifically pain management (Peterson et al. and Rawlins et al.), PTSD (van Veelen et al.), and trauma (Greenhalgh et al. and Lombardo et al.). But there were also some surprises. Van Veelen's idea of using VR to enable variable levels of immersion in PTSD therapy would appear to give therapists another tool that could only be possible in VR (van Veelen et al.). Similarly, Appel uses VR for relaxation therapy in veterans with dementia, in which there is a higher-than-average occurrence (Appel et al.; Appel et al.). There is also a heightened awareness of the importance of usability of VR/ XR systems (see Greenhalgh et al. and Rawlins et al.).

We were also surprised by what we did not see. We anticipated more training topics. Lombardo presents a VR trauma simulator for education and training (Lombardo et al.), but it is an unconventional training study where the impact of the training system is measured in skill transfer to the real world. Which brings us to an important point on the current state of knowledge about VR/XR in medicine and healthcare.

A common shortcoming in VR/XR research across all domains, and extending over the past 25 years to the first VR "wave", has been our inability to identify the characteristics of a problem for which VR/XR is well-suited, and then identify how to apply it to achieve the best possible outcome. Consider, as an analogous example, the graphical user interface. Experimentation in the 1990's resulted in GUI standards in use today that allow UX designers to create effective interfaces without the need for new empirical studies to prove it.

A reasonable goal for our community is to enumerate the types of tasks that have been performed and/or trained using VR/XR. We then decompose those tasks into component parts to identify how one task is analogous or distinct from other tasks. We know how to do this; this is task analysis. How 
is a surgical probing task similar and/or different from a field medic tourniquet trainer? Clearly these tasks are different, yet they have similarities. Both require two hands. Both involve fine and gross motor skills. Do these similarities suggest features of a surgical trainer that could inform the design of a trainer for the field medic?

Such a framework could allow for the explosive growth of knowledge because point solutions could finally be connected through task performance rather than by the apparatus. Technology change is inevitable. Tasks change slower, but they do change as new medical procedures and techniques are developed. User attributes are persistent.

This persistence is heralding a shift into a new phase which builds upon the established foundation of VR's basic science. Partly due to the pandemic, partly due to price reductions, heightened user demand and user sophistication require devotion to the practical science of implementation. More people, across all levels of the organization, are more accepting of and knowledgeable about VR. The growing list of implementation considerations testifies to the authors' resilience. They have pioneered into this frontier and have come back to provide us with answers and even more questions.

Infection prevention has rightly called our attention to user safety. In the past, we could casually pass a headset between host and user. Now, we plan hosting sessions around the time, equipment and materials required to guarantee that we are not causing harm. This novel issue has been a barrier, and at a minimum it has slowed organizational adoption and stalled research projects. At the same time, we will emerge with safer users and deeper appreciation for the benefits VR brings.

As the number of users expands, a new hybrid group is emerging. They are not end-users per se; they represent a meta-user role. A good example of this is a psychologist who provides prolonged exposure therapy. When this provider adds VR Exposure Therapy to traditional treatment, the psychologist is a user of the VR interface. The patient is also an end-user: the psychologist and the patient work together to use the technology to enhance patient outcomes. Hence, the psychologist is both hosting the VR session and providing therapeutic care. The technological competency, motivation, and attitude toward technology of this provider will have a strong impact on quality of the end-user's overall experience. From a humancentered design perspective, this user-provider group must be included in the design process early on. Two papers shed light on this user-provider group (Appel et al.; Greenhalgh et al.).

Regarding gatekeepers and user-providers, there can be resistance to the introduction of technology. Sometimes, AI and robotics can appear as threats, competitors with the human professionals who are the heart of every healthcare system. As VR applications grow, rather than de-humanizing connections, user-providers may find that the use of technology may actually enhance the quality of interpersonal connection between patient and provider (Rawlins et al.).

Hosting VR sessions is an essential role. However, as VR continues to move away from brick-and-mortar medical facilities to in-home access, the appearance of hosting is changing. Greenhalgh et al. remind us that the design of VR applications targeted for the healthcare field in general, and telehealth applications specifically, demand attention to elements beyond discoverability and intuitiveness. We must also ensure that we mitigate the possibilities of dizziness, motion sickness and falls. Though these issues have always been present in VR, we have traditionally been able to rely upon the physical presence of a host who wears many vitally important hats: safety officer, proctor, interface educator, care provider. As we venture into telehealth, the no-host design goal becomes a primary design criterion.

Another emerging user-focused advent is the progress toward a more precise understanding of when to introduce VR into a traditional treatment plan for an individual patient. Being more precise when matching specific patients to VR will help focus the technology and yield more potent effects. The two distinct care situations (scheduled and targeted) identified by Appel et al.'s two papers illustrate another form of identifying ripe opportunities for introducing VR (Appel et al.; Appel et al.). Similarly, Van Veelen et al. advocate for customization of the VR experience to more precisely match VR to an individual (van Veelen et al.).

We congratulate the authors for their perseverance. The momentum of VR incorporation grows every year. Let's convene again in a year or two and see what new answers and questions we can discuss.

\section{AUTHOR CONTRIBUTIONS}

Each of the authors was a co-editor of this issue and was the primary editor responsible for a subset of the papers accepted for publication. Each editor contributed text to the editorial article associated with the papers for which they were responsible.

Conflict of Interest: The authors declare that the research was conducted in the absence of any commercial or financial relationships that could be construed as a potential conflict of interest.

Publisher's Note: All claims expressed in this article are solely those of the authors and do not necessarily represent those of their affiliated organizations, or those of the publisher, the editors and the reviewers. Any product that may be evaluated in this article, or claim that may be made by its manufacturer, is not guaranteed or endorsed by the publisher.

Copyright (c) 2022 Darken, Peterson and Gore. This is an open-access article distributed under the terms of the Creative Commons Attribution License (CC $B Y)$. The use, distribution or reproduction in other forums is permitted, provided the original author(s) and the copyright owner(s) are credited and that the original publication in this journal is cited, in accordance with accepted academic practice. No use, distribution or reproduction is permitted which does not comply with these terms. 